\title{
Editorial
}

\section{Radar Space-Time Adaptive Processing}

\author{
Fabian D. Lapierre, ${ }^{1}$ Jacques G. Verly, ${ }^{2}$ Braham Himed, ${ }^{3}$ Richard Klemm, ${ }^{4}$ and Marc Lesturgie ${ }^{5}$ \\ ${ }^{1}$ Department of Electrical Engineering, Royal Military Academy, 1000 Brussels, Belgium \\ ${ }^{2}$ Department of Electrical Engineering and Computer Science, University of Liège, 4000 Liège, Belgium \\ ${ }^{3}$ Air Force Research Laboratory, Rome, NY 13441, USA \\ ${ }^{4}$ FGAN-FFM, 53343 Wachtberg, Germany \\ ${ }^{5}$ ONERA/DEMR, 91761 Palaiseau, France
}

Received 29 December 2005; Accepted 29 December 2005

Copyright ( 2006 Fabian D. Lapierre et al. This is an open access article distributed under the Creative Commons Attribution License, which permits unrestricted use, distribution, and reproduction in any medium, provided the original work is properly cited.

Space-time adaptive processing (STAP) is a signal processing technique that was originally developed for detecting slowmoving targets using airborne radars. The general principle of STAP is as follows. The radar transmits a train of $M$ coherent pulses. The echoes from potential targets (and clutter) are collected at each of the $N$ elements of an antenna array. Separate receiver chains are attached to each of the array elements. The received signals are sampled at a series of $L$ successive ranges (i.e., distances) also referred to as range gates. STAP processing is applied to the $M \times N$ matrix of samples collected at each such range. This matrix is typically called a snapshot. The ensemble of snapshots at all successive ranges is referred to as a data cube and contains all the information available for target detection within a coherent processing interval (CPI). If the radar transmitter and receiver are located on the same platform (airplane or satellite), the configuration is called a monostatic configuration. If not, the term "bistatic" is used. In bistatic configurations, the carrying platforms are not only distinct, but they can also move independently.

Although the general principles of STAP have been known since at least the 1980's, the field has seen a major regain of interest in the 1990's, mainly as a result of the significant increase in computational power. Much of the 1990's focused on three major topics of interest. The first is the application of STAP to monostatic radar platforms. The second is the design of computationally efficient adaptive methods (suboptimum methods) to reduce the computational load of the STAP processor. The third is the design of methods to mitigate barrage jammers. Throughout this period of time, investigators focused almost exclusively on uniform linear arrays (ULAs), where the elements are on a line and uniformly spaced.
More recently, much of the attention in STAP has shifted to a new series of issues, which are now briefly described.

(1) There is significant interest in bistatic configurations for the simple reason that they allow the receiving platform to remain covert during operation.

(2) Researchers are considering arrays that go beyond ULAs, such as arbitrary 3D antenna arrays. One particular case of three-dimensional (3D) array is the conformal antenna array (CAA) that follows the surface of the carrying platform, such as the fuselage of an airplane or the side of a balloon.

(3) There is a growing need for STAP to perform well in heterogeneous environments. This problem refers to the lack of (wide-sense) stationarity of the received signals with respect to range. Stationarity tends to disappear in bistatic configurations or when antennas other than ULAs are used. Once the hypothesis of stationarity is no longer verified, conventional covariance estimation methods can no longer be used. Stationarity also tends to disappear when terrain deviates from being flat with uniform reflectivity properties and in the presence of internal clutter motion such as tree leaves moving in the wind.

(4) The problems just mentioned have given rise to methods known as knowledge-aided STAP, which attempt to remove as much of the heterogeneity from the snapshots prior to using conventional estimation methods. This is done by using a priori knowledge, typically stored in databases. Knowledge-aided STAP falls in the general domain of knowledge-aided signal processing.

(5) Finally, STAP techniques are currently moving into new areas such as sonar and telecommunications, and also in new application areas such as the detection of plastic landmines. 
The goal of this special issue is to discuss the state of the art in radar STAP techniques (suboptimal, bistatic, etc.) and to explain why STAP techniques are also proving useful in domains that were probably not initially anticipated.

\section{Cancellation of barrage jammers}

Jamming remains a significant problem in monostatic STAP. This is particularly true of barrage jammers, which emit jamming signals with very wide bandwidths. In monostatic configurations, the receiver is colocated with the transmitter and is thus easily located and jammed.

In the present discussion, only a single jammer is considered for simplicity. Classical jammer suppression techniques use spatially adaptive processing to remove the jamming signal from the received signal. In other words, no processing is done along the time dimension, whether fast-time or slowtime. This technique is effective as long as the target and jammer are sufficiently separated in angle and do not both fall within the mainbeam of the receive antenna. In the limit, when the target and jammer are aligned, the spatially adaptive processor cannot cancel the jammer.

An emerging class of space-time processing techniques, which may be referred to as space fast-time adaptive processing, can overcome this problem by processing in the fasttime dimension. Fast-time processing differs from more traditional slow-time processing in the following way. If a train of pulses is transmitted, one can process simultaneously the echoes from all these pulses at a particular range. This is slow-time processing. In fast-time processing, one processes simultaneously the echoes corresponding to each particular pulse and to several ranges, typically located in the vicinity of the range being interrogated. The combination of space processing with slow-time or fast-time processing leads to space slow-time processing and to space fast-time processing, respectively. Successful fast-time processing is contingent upon the availability of coherent multipath in the form of terrainscattered interference (TSI).

The paper by Y. Seliktar, D. B. Williams, and E. J. Holder presents a method for space fast-time monopulse processing that can provide better estimation of the jammer's angle than classical spatially adaptive monopulse can. This method also exploits the presence of TSI. The capabilities of the method are illustrated using the mountaintop data, which contains one jammer as well as TSI. The approach is shown to perform significantly better than conventional monopulse and spatially adaptive monopulse.

The paper by D. Madurasinghe and A. P. Shaw addresses the computational complexity of a space fast-time adaptive processor that uses the TSI to cancel barrage jammers. Recall that, in fast-time processing, one piles up a large number of echoes coming from different ranges. Since there is typically a large number of ranges and since the time interval between two consecutive echoes is very short, it becomes virtually impossible to process this large amount of data in real-time. This problem is solved by introducing a preprocessor that allows the STAP processor to select only two desired range returns to form the space fast-time snapshot. The main contribution of the paper is the design of a new space fast-time adaptive processor relying on (eigenvectorbased) super-resolution, which also has the feature of being extremely fast.

\section{Knowledge-aided processing}

In a classical STAP processor, the presence of heterogeneities arising from the use of an arbitrary antenna array and the presence of internal clutter motion (ICM), can lead to severe performance degradation. The goal of the knowledge-aided sensor signal processing and expert reasoning (KASSPER) program, initiated by the Defense Advanced Research Projects Agency (DARPA), is to develop new robust techniques that are able to detect and track targets that are either stationary or moving in the presence of heterogeneities. This is typically achieved by providing auxiliary information, such as digital elevation models (DEMs), clutter reflectivity maps, and GPS positions, to the detection and tracking systems.

The paper by J. S. Bergin and P. M. Techau explores signal processing techniques based on a mix of ground moving target indicator (GMTI) processing and synthetic aperture radar (SAR) processing. Whereas STAP aims at detecting slow-moving targets using a short CPI, SAR aims at detecting stationary targets with long CPIs. The authors focus here on STAP implementations using long GMTI CPIs as well as SAR-like processing strategies for detecting targets that move very slowly. SAR data is then used as an aid to improve target detection. The processing technique proposed includes SAR-derived knowledge-aided constraints to improve detection performance in an environment that includes large discrete scatterers, which are responsible for increased false alarm rates. The SAR imagery is, for example, used to locate strong clutter discretes.

The paper by D. Page and G. Owirka describes knowledge-aided STAP (KA-STAP) techniques that use data corresponding to several independent CPIs. This can prove useful in surveillance scenarios where the ground may contribute returns extending over multiple CPIs. The paper shows how data coming from multiple CPIs can be used to enhance the detection performance of the STAP processor. This data is used to enhance the accuracy of the estimated clutter reflectivity maps and, thus, to provide improved knowledge about clutter statistics in nonhomogeneous terrain environments. These maps are estimated using the data recorded over multiple CPIs, DEMs, and geo-registration of the clutter scatterers. This registration is needed since the position of the moving platform varies from one CPI to the next. The reflectivity maps are used to predict the clutter covariance matrices as a function of range. The techniques of covariance tapering, adaptive estimation of gain and phase corrections, knowledge-aided prewhitening, and eigenvalue scaling are also exploited to estimate the space-time filter needed to reject colored interference. This filter cannot handle clutter discretes, but a technique for suppressing large discrete returns is proposed in the paper. Simulation results show that, compared to standard STAP processing, the proposed method leads to more than an order of magnitude in false alarm rate reduction. 


\section{Landmine detection}

One approach for detecting buried plastic landmines is to use quadrupole resonance (QR) based techniques. However, the frequency of the emitted QR signal is located within the $A M$ radio frequency band. The received signal may thus be corrupted by strong radio-frequency interference (RFI). The challenge is to mitigate the RFI in the received signal to be able to extract the very weak signal characterizing the landmine. If the signal is received by an antenna array, the spatial correlation of the signal can be used to improve the rejection of these RFIs. However, just exploiting the spatial correlation does not lead to a good detection probability. At first sight, it may come as a surprise that STAP could help in this application, since STAP is typically used to detect slow-moving targets, whereas landmines are typically not moving. The connection is the following. It turns out that the temporal variations of the QR echoes from pulse to pulse is a signature of the chemical present inside the mines, such as trinitrotoluene (TNT) and royal demolition explosive (RDX). The QR echoes are thus both spatially and temporally correlated. Therefore, STAP processing should help reject the RFIs by exploiting these correlations.

The paper by G. Liu, Y. Jiang, H. Xiong, J. Li, and G. A. Barrall exploits the spatio-temporal correlation of the RFIs to improve the detection of TNT, which leads to a better landmine detection performance. The authors propose three distinct detection methods, which are later combined. The first method exploits only the spatial correlation of the RFIs by using an antenna array. A maximum-likelihood (ML) estimator and a constant false alarm rate (CFAR) detector for TNT detection are also proposed. The second method adopts a multichannel autoregressive (MAR) model to take into account the temporal correlation of the RFIs and leads to a detector based on this model. The third method improves RFI mitigation by using a two-dimensional robust Capon beamformer (RCB) together with an ML estimator. Finally, the three methods are exploited jointly to improve detection performance. Experiments using real data demonstrate the soundness of the proposed RFI mitigation methods and of the combined approach.

\section{Fabian D. Lapierre Jacques G. Verly Braham Himed Richard Klemm Marc Lesturgie}

Fabian D. Lapierre was born in Huy, Belgium. He received the Ingénieur Electronicien degree from the University of Liège, Belgium, in 2000. In 2004, thanks to a fellowship of the F.N.R.S. (Fond National de la Recherche Scientifique), Brussels, Belgium, he received his Ph.D. degree from the University of Liège, Belgium. He is currently, a Member of the Electrical Engineering Department of the Royal Military Academy in Brussels, Belgium. His research interests are mainly focussed on space-time adaptive processing (STAP) and on the simulation of infrared target signatures.

Jacques G. Verly was born in Liège, Belgium. He received the Ingénieur Electronicien degree from the University of Liège, Belgium, in 1975. Through a sponsorship of the Belgian American Educational Foundation (BAEF), he attended Stanford University, Stanford, Calif, where he received the M.S. and Ph.D. degrees in electrical engineering in 1976 and 1980, respectively. From 1980 to 2000, he was at MIT Lincoln

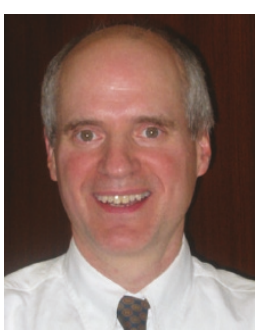
Laboratory, Lexington, Mass, where he carried out research in several different areas, including image processing and computer vision for a variety of imaging sensors, such as visible, laser radar, fully polarimetric SAR, and IR. Since 2000, he has been a Professor in the Department of Electrical Engineering and Computer Science (also known as the "Institut Montefiore") of the University of Liège, Belgium. He is a Founder of the Signal and Image Exploitation Research Unit (INTELSIG). His current research interests are principally in medical imaging (image-guided surgery), radar signal processing (space-time adaptive processing), and object tracking in video streams (for video surveillance and sports analysis). He has about 170 publications and 2 US patents. He is a CRB Fellow of the Belgian American Educational Foundation.

Braham Himed received his B.S. degree in electrical engineering from Ecole Nationale Polytechnique of Algiers in 1984, his M.S. and Ph.D. degrees, both in electrical engineering, from Syracuse University, in 1987 and 1990, respectively. From 1990 to 1991, he was an Assistant Professor in the Electrical Engineering Department at Syracuse University. In 1991 he joined Adaptive Technology, Inc., Syracuse, NY, where he was re-

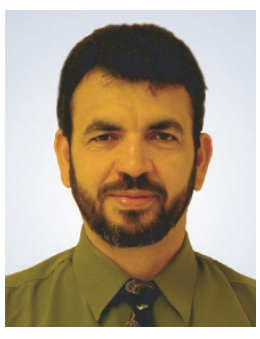
sponsible for several radar systems analyses. In 1994 he joined Research Associates for Defense Conversion, Marcy, NY, where he was responsible for radar systems analyses and signal processing studies. Since March 1999, he is with the U.S. Air Force Research Laboratory, Sensors Directorate, Radar Signal Processing Branch, Rome, NY, where he is involved with several aspects of airborne and spaceborne phased array radar systems. His research interests include detection, estimation, multichannel adaptive signal processing, time series analyses, array processing, space-time adaptive processing, hot clutter mitigation, and ground penetrating radar technology. Dr. Himed is the recipient of the 2001 IEEE region award for his work on bistatic radar systems. Since 1993, he has also been an Adjunct Professor at Syracuse University. Dr. Himed is a Senior Member of the IEEE and a Member of the Radar Systems Panel.

Richard Klemm received his Dipl. Ing. and Dr. Ing. degrees in communications from the University of Technology in Berlin, Germany, in 1968 and 1974, respectively. He is a Senior Scientist at FGAN (The German Defense Research Establishment), where he has been doing research on various aspects of radar signal processing. His numerous publications include a book on space-time adaptive processing whose $3 \mathrm{rd}$ edition will appear soon. He has also been an editor of a book on Applications of Space-Time Adaptive Processing including chapters by 45

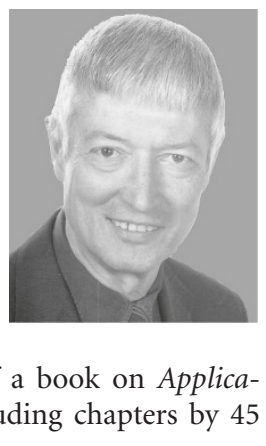


renowned experts worldwide. He has given numerous seminars on invitation by different countries and organizations. He organized and chaired various scientific conferences. In 1996, he initiated the European Conference on Synthetic Aperture Radar (EUSAR). He received several awards in recognition of his work.

Marc Lesturgie was born in Rouen (France) in 1963. He graduated from ENSAE (Ecole Nationale Supérieure de l'Aéronautique et de l'Espace, Toulouse) in 1985, and obtained a DEA (Master's degree) in electronics and microwaves from the University of Toulouse in 1986. In 1987, he joined the French research center ONERA (Office National d'Etudes et de Recherches Aérospatiales) as a Research Engineer in the

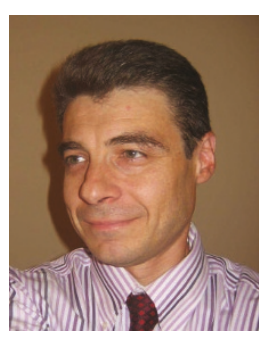
area of low-frequency radar. Head of the "Advanced Radar Concepts" team from 1996 to 2000, he is currently in charge of the prospective in the area of electromagnetic detection, including any application of monostatic or bistatic radar. Since 2004, he has been the Technical Manager of SONDRA, a joint laboratory established between France (ONERA and Supelec) and Singapore. Chairman of the SEE-Committee 23 (radio-location and navigation), Lecturer in several French and international universities and engineering schools for more than 15 years, he was also the Chairman of the Program Committee for the last International Radar Conference held in France (Radar 2004). 